\title{
Fine mapping of rice bacterial leaf blight resistance loci to major Korean races of Xoo (Xanthomonas oryzae) and development markers
}

Lee Myung Chul*, Choi Yu-Mi, Yoon Hyemyeong, Lee Sukueung, Yoon-Hyun Do, Oh Sejong

National Agrobiodiversity Center, National Institute of Agricultural Sciences, RDA, Republic of Korea *e-mail:mcleekor@korea.kr

Bacterial leaf blight (BLB), caused by X. oryzae pv. oryzae (Xoo), is one of the most destructive diseases of rice due to its high epidemic potential. Understanding BLB resistance at a genetic level is important to further improve the rice breeding that provides one of the best approaches to control BLB disease. In the present investigation, a collection of 192 accessions was used in the genome-wide association study (GWAS) for BLB resistance loci against four Korean races of Xoo that were represented by the prevailing BLB isolates under Xoo differential system. A total of 192 accessions of rice germplasm were selected on the basis of the bioassay using four isolated races of Xoo such as K1 and K2. The selected accessions was used to prepare 384-plex genotyping by sequencing (GBS) libraries and Illumina HiSeq 2000 paired-end read was used for GBS sequencing. GWAS was conducted using TASSEL 5.0. The TASSEL program uses a mixed linear model (MLM). The results of the bioassay using a selected set of 192 accessions showed that a large number of accessions $(93.75 \%)$ were resistant to K1 race and K2 resistant germplasm proportion remained between 66.67. The genotypic data produced SNP matrix for a total of 293,379 SNPs. After imputation the missing data was removed, which exhibited 34,724 SNPs for association analysis. GWAS results showed strong signals of association at a threshold of $[-\log 10(P$-value $)]$ more than 5 (K1 and K2) for nine of the 39 SNPs, which are plausible candidate loci of resistance genes. These SNP loci were positioned on rice chromosome 2, 9, and 11 for K1 and K2 races. The significant loci detected have also been illustrated and make the CPAS markers for $N B S-L R R$ type disease resistance protein, SNARE domain containing protein, Histone deacetylase 19, NADP-dependent oxidoreductase, and other expressed and unknown proteins. Our results provide a better understanding of the distribution of genetic variation of BLB resistance to Korean pathogen races and breeding of resistant rice. 